\title{
Standardization of an Indirect ELISA for Serological Diagnosis of Aspergilloma
}

\author{
Nariman Shah-Hosseini ${ }^{1,{ }^{*}}$, Alireza Khabiri ${ }^{2}$, Farideh Bagheri ${ }^{2}$, Hashem Yazdi $^{2}$, Nima Farajianfar $^{2}$ \\ ${ }_{2}^{1}$ Islamic Azad University, Lahijan Branch, Faculty of Science, Department of Microbiology, Lahijan, IR Iran \\ 2 Pasteur Institute of Iran, Department of Mycology, Tehran, IR Iran \\ *Corresponding author: Nariman Shah-Hosseini, Islamic Azad University, Lahijan Branch, Faculty of Science, Department of Microbiology, Lahijan, IR Iran. Tel.: +98-1412229081, Fax: \\ +98-1412229081, E-mail: Nariman.shahhosseini@yahoo.com.
}

Received: May 18, 2012; Revised: May 29, 2012; Accepted: Jun 01, 2012

Background: So far several literatures have discussed that GMP as a major cell wall component in Aspergillus species and can also be secreted into the medium as a component of the exoantigen. Moreover, one of the critical points in designing an ELISA is the preparation of the antigen.

Objectives: The main aim of this study was the identification of GMP and standardization of an indirect ELISA for the serological diagnosis of Aspergilloma.

Materials and Methods: An indirect enzyme linked immunoassay (ELISA) was developed and standardized for the serological diagnosis of Aspergilloma.

Results: Among 500 serum samples, 35 had positive and 465had negative ELISA test against Aspergilloma. In comparison, serum samples were tested by immunoblotting test, considered as the standard. In relation to the immunoblotting, the ELISA presented $94.2 \%$ sensitivity, 99.5\% specificity, $94.2 \%$ positive predictive value, $99.5 \%$ negative predictive value and $99.2 \%$ precision.

Conclusions: It was concluded that the ELISA is a suitable method for large scale screening of antibodies against Aspergilloma. The easy techniques have shortened the required time of tests (approximately 1.30 hours for our ELISA, as opposed to 2.30 hours with commercial ELISA kit). This may be a substantial benefit for large numbers of samples that should be tested.

Keywords: Serologic Tests; Enzyme-Linked Immunosorbent Assay; Immunoblotting

\section{Background}

Aspergillus spp are ubiquitous opportunistic molds that cause invasive syndromes. The small size of conidia are present in the air at concentration 1 to 100 conidia $/ \mathrm{m}^{3}$. Human inhaled several hundred conidia of Aspergillus per day, but it rarely resulted in disease in immunocompetent persons, since these conidia are easily eliminated $(1,2)$. The genus Aspergillus includes over 185 species. Around 40 species have so far been reported as causative agents of opportunistic infections in human and animals. The frequency and relative importance of these infections are on the rise in all developed countries, which is possibly related to the increased numbers of immunocompromised patients $(3,4)$.

Glactomannoprotein (GMP) is an essential molecule in pathogenic Aspergillus fumigatus. GMP is also produced in the host and is a well-known A. fumigatus antigen. Antibodies directed against this antigen have been detected in infected patients with aspergilloma. In addition, it has been repeatedly shown that this molecule circulates in the biological fluids of patients with invasive aspergillosis. Serological diagnosis of this life-threatening fungal infection remains based on the detection of GMP in the serum, urine, or bronchoalveolar lavage samples of infected patients $(5,6)$.

\section{Objectives}

The main aim of this study was the identification of GMP and standardization of an indirect ELISA for the serological diagnosis of Aspergilloma.

\section{Materials and Methods}

\subsection{Strain and Standard Culture Conditions}

A. fumigatus strain AF-293 was cultivated in Sabouraud liquid medium ( $2 \%$ glucose, $1 \%$ peptone adjusted to $\mathrm{pH}$ 6.1). Culture was incubated at $37^{\circ} \mathrm{C}$ on a rotary shaker $150 \mathrm{rpm}, 0.5$ volume of air/volume of medium/min for 3 days (7).

Implication for health policy/practice/research/medical education:

This study was conducted to compensate the need for importation of ELISA kit for serological diagnosis of Aspergilloma in Iran.

Copyright (C) 2012, Shahid Beheshti University of Medical Sciencces;. This is an Open Access article distributed under the terms of the Creative Commons Attribution License (http://creativecommons.org/licenses/by/3.0), which permits unrestricted use, distribution, and reproduction in any medium, provided the original work is properly cited. 


\subsection{Antigen Precipitation}

The culture filtrates were suspended with 4 volumes of $100 \%$ ethanol at $25^{\circ} \mathrm{C}, \mathrm{rpm} 150$, overnight. The solutions were precipitated using centrifugation at $4000 \mathrm{~g}$ for 30 minutes. The ethanol precipitates (EP), washed three times with ethanol, were re-suspended in PBS (Phosphate buffer saline) and stored at $4^{\circ} \mathrm{C}(8)$.

\subsection{Galactomannoproteins (GMP) Analysis}

GMP sample was analyzed by sodium dodecyl sulfate polyacrylamide gel electrophoresis (SDS-PAGE) using $4 \%$ staking and 10\% separating gels. The GMP samples were mixed with the $\beta$-mercaptoethanol and SDS loading buffer (50 mM Tris-HCl, 2\% [w/v] SDS, pH 6.8) and subjected to electrophoresis at $140 \mathrm{~V}$ for 1 hour. After electrophoresis, GMPs were stained with silver staining technique or electro transferred to nitrocellulose membrane for 1 hour at $12 \mathrm{~V}$ in Tris $\mathrm{HCl}$ buffer ( $\mathrm{pH}$ 8.6). After blocking, nitrocellulose membrane was immersed in the pool of periodate $0.1 \mathrm{M}$ for 1 hour, then it was rinsed three times with water. Afterwards, the bands labeled with proxidase-conjugated antibodies diluted at 1:5000. The binding verified with 3,3'-Diamonibenzidine tetra-Hydrochloride (DAB) as the substrate (9).

\subsection{ELISA Procedure}

In brief, the plates were coated overnight at $4^{\circ} \mathrm{C}$ with $10 \mathrm{~mL} /$ well of an appropriate dilution of GMP as antigen (dilution 1:100). Plates were either used the next day or stored at $-20^{\circ} \mathrm{C}$ for further use. The plates were washed three times with washing buffer (PBS 0.05\% Tween 20), before the use. Subsequently, the washing buffer removed and an appropriate dilution of each test serum (1:20) was added to each of two wells. After an incubation period of 30 minutes at $37^{\circ} \mathrm{C}$, the plates were washed three times as mentioned above and $100 \mathrm{~mL}$ of an appropriate dilution (typically 1:1000) of conjugated antibodies were added to each well. Following incubation for 30 minutes at $37^{\circ} \mathrm{C}$, the plates were again washed three times and $100 \mathrm{~mL}$ of TMB was added. After 15 minutes (previously determined as the optimum time for stopping), the reaction was stopped by adding $50 \mathrm{~mL} \mathrm{H} 2 \mathrm{SO} 4$ to each well. Plates were read on an ELISA reader at an optical density of $450 \mathrm{~nm}$.

\subsection{Serum Panels}

Serum samples from 500 suspected patients for aspergilloma infection, provided by microbiology laboratories from different hospitals during February 2010 to April 2011, were used in this study. Sera of patients were analyzed for the presence of antibody against $A$. fumigatus GMP using an indirect enzyme-linked immunosorbent assay (ELISA).

\subsection{Calculation of the Cut-Off Point}

The cut-off point was calculated based on the mean OD of the negative sera, and three standard deviations (cutoff point $=$ mean $\mathrm{OD}+3$ standard deviations).

\subsection{Comparison With a Commercially Available ELISA}

The ELISA kit and a commercially available ELISA kit (IBL) were compared. The commercial test was performed as recommended by the manufacturer, with 500 serum samples for antibodies against A. fumigatus. The obtained results of both ELISA kits were calculated in relation to the immunoblotting and compared.

\section{Results}

\subsection{Identification of GMPs}

GMPs are primary metabolites that are released in the culture medium of A. fumigatus . Previous studies have demonstrated that GMP is a glycoprotein with a molecular weight between 55 - $60 \mathrm{kDa}(9)$. The analysis of ethanol precipitation (EP) on a 10\% SDS-PAGE gel silver stained and electro transferred to nitrocellulose membrane indicated that GMPs is $60 \mathrm{kDa}$ (Figure 1 ).

Figure 1. Analysis of Ethanol Precipitation (EP), Stored at $37{ }^{\circ} \mathrm{C}$, and $-20{ }^{\circ} \mathrm{C}$ (Lane 1 and 2)

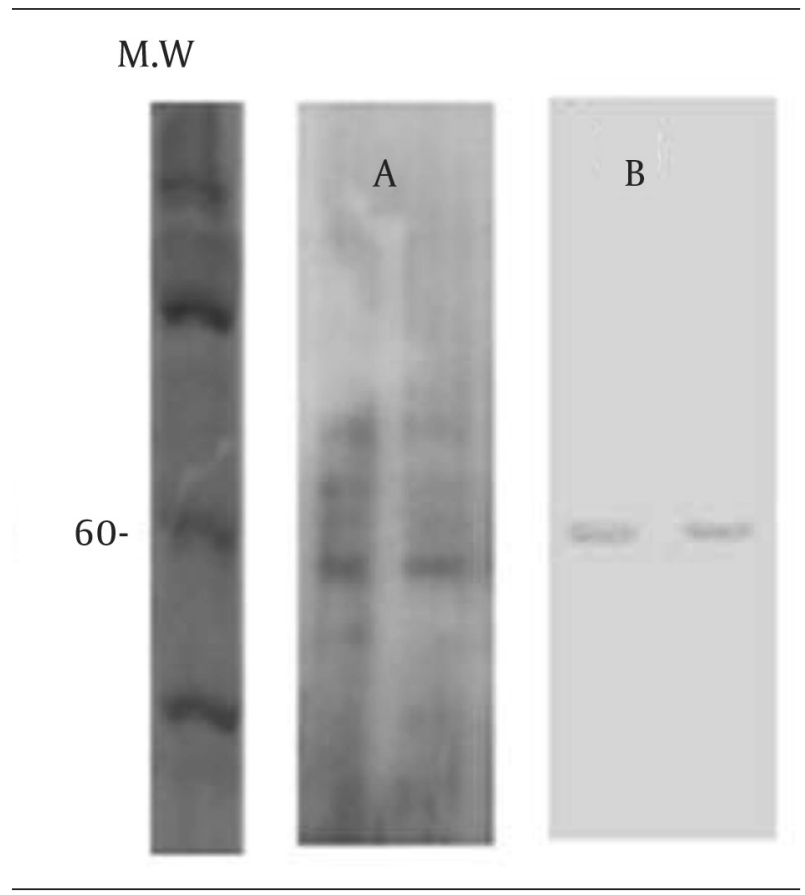

A) On a $10 \%$ separating gel silver stained B) and electro transferred to nitrocellulose membrane 


\subsection{Performance of ELISA}

Antigen, conjugated antibodies, serum dilutions and the timing were optimized based on the dilutions where the distinction between positive and negative sera was most evident. Between 500 suspected patients' sera, 35 were serologically positive. The same results were obtained when compared with a commercial ELISA kit. The differences of OD observed in the positive and negative samples were quite marked.

\subsection{Determination of the Cut-Off Point}

The average of the OD obtained from 465 antibody-negative sera, was 0.178. Serum samples with an OD above the cut-off point were considered as positive and sera with OD values below the cut-off point were considered as negative. All samples with ODs close to the cut-off point were considered as suspect and were retested.

Cut-off point $=\mathrm{m} \mathrm{OD}+3 \mathrm{~s}$.

Cut-off point $=0.109+(3 \times 0.023)$

Cut-off point $=0.178$

\subsection{ELISA Data Were Confirmed by Immunoblot- ting (Western Blot) Results}

Since the immunoblotting is an efficient method, the sera were also tested by immunoblotting. Among 35 sera samples which were positive using ELISA, 33 were positive using immunoblotting. Thus, the ELISA test was found sensitive and specific (Table 1 ).

Table 1. Comparison of the Results Obtained With Indirect ELISA and the Immunoblotting Assay on 500 Serum Samples and Analysis of Validity of the Test

\begin{tabular}{|c|c|c|}
\hline \multicolumn{3}{|c|}{ Immunoblotting ${ }^{a}$} \\
\hline ELISA & Positive & Negative \\
\hline 33 & $33(a)$ & $2(b)$ \\
\hline 2 & $2(\mathrm{c})$ & $465(d)$ \\
\hline \multicolumn{3}{|c|}{$\begin{array}{l}\text { a Analysis of validity of the ELISA (based on the Immuno blotting } \\
\text { results). Sensitivity }(a / a+c) \times, 100=33 / 33+2 \times 100=94.2 \% \text {. Specificity } \\
(d / b+d) \times 100=465 / 2+465 \times 100=99.5 \% \text {. The negative predictive value } \\
(d / c+d) \times 100=465 / 2+465 \times 100=99.5 \% \text {. Positive predictive value }(a / a \\
+b) \times 100=33 / 33+2 \times 100=94.2 \% \text {. Precision }(a+d / a+b+c+d)=33+ \\
465 / 33+2+2+465 \times 100=99.2 \%\end{array}$} \\
\hline
\end{tabular}

\subsection{Comparison of tested ELISA and Immunoblot- ting and the Analysis of Test Validity}

In Table 1 the results of the comparison between the ELISA and the Immunoblotting are shown. The analysis of the validity of the test is at the footnotes in Table 1.

\section{Discussion}

The ranges of Aspergillus infections that may be acquired by individuals include: aspergilloma, allergic bronchopulmonary aspergillosis, invasive aspergillosis, sinusitis, otomycosis, ocular infections, CNS infection, osteomyelitis, cutaneous aspergillosis, endocarditis, urinary tract infection (10). As a result, a rapid and reliable diagnosis test can play a critical role in the beginning of specific treatment and improvement of the patient survival quality. The presumptive diagnosis of aspergilloma is made by imaging, but the definite criteria for diagnosis rely on clinical and laboratory data. The common clinical signs of aspergilloma are chronic cough (the majority of patients have hemoptysis), malaise and weight loss. Specified laboratory tests, serological techniques based on detection of antibodies specific to an antigenic cell wall galactomannoprotein of A. fumigatus are reliable (11).

So far several literatures have discussed that GMP as a major cell wall component in Aspergillus species and can also be secreted into the medium as a component of the exoantigen $(8,12)$. Moreover, one of the critical points in designing an ELISA is the preparation of the antigen. The described method in this study, for the preparation of the ELISA antigen was highly effective, since it provided a good discriminative capacity between antibody-positive and negative samples. In this study, we described the standardization of an indirect ELISA test for the serological diagnosis of Aspergilloma. The indirect ELISA was designed for the large scale detection of antibodies to $A$. fumigatus. The test was found very practical and simple to perform, providing in most cases a clear distinction between positive and negative sera. Most positive and negative sera could actually be identified visually; only in a few cases visual inspection was not sufficient to distinguish the differences between positive and negative samples, as immediately confirmed by OD analysis.

The sensitivity, specificity, positive and negative predictive values, and precision calculated for ELISA in comparison with the Immunoblotting test, adopted as the golden standard. The easy method has shortened the required time of the test (approximately 1.30 hours for our ELISA kit, as opposed to 2.30 hours with others). This may be a substantial benefit when large numbers of samples should be tested. In addition, the test was shown to be very much relied on the repetitions led to the same (positive or negative) results. The sensitivity and specificity of the ELISA test is certainly related to the cut-off point (13). The cut off was determined based on the mean OD of the actual negative population of sera. In order to establish a direct comparison with a widely used, commercially available ELISA, our ELISA kit was compared with the ELISA-IBL. In comparison, IBL ELISA presented slightly more sensitivity (95\%) than the tested ELISA (94.2\%). However, the specificity of our ELISA was slightly higher (99.5\%) than the IBL ELISA (95\%). A growing number of enzyme immunoassays for the serological diagnosis of Aspergilloma are being marketed around the world. However, to date, none of the diagnostic kits which are available for the serodiagnosis of Aspergilloma are produced in Iran, which has increased the need for importations, thus sub- 
stantially increased the costs of serological tests. In order to find an alternative to the imported kits, we designed an ELISA kit. The test showed an adequate performance compared to the Immunoblotting assay.

\section{Acknowledgements}

The author is extremely grateful to Pasteur Institute of Iran for the financial support to fulfill this research.

\section{Authors' Contribution}

Study concept and design: Alireza Khabiri, Farideh Bagheri, Nariman Shah-Hosseini. Analysis and interpretation of data: Nariman Shah-Hosseini, Alireza Khabiri, farideh Bagheri, Hashem Yazdi and Nima Farajianfar. Drafting of the manuscript: Nariman Shah-Hosseini. Critical revision of the manuscript for important intellectual contents: Nariman Shah-Hosseini. Statistical analysis: Nariman Shah-Hosseini.

\section{Financial Disclosure}

We declare that there is no conflict of interest.

\section{Funding/Support}

This study was financially supported by Pasteur Institute of Iran.

\section{References}

1. O'Gorman CM, Fuller HT, Dyer PS. Discovery of a sexual cycle in the opportunistic fungal pathogen Aspergillus fumigatus. $\mathrm{Na}$ - ture. 2008;457(7228):471-4.

2. Segal BH. Aspergillosis. N Engl J Med. 2009;360(18):1870-84.

3. Verweij PE, Brandt ME, Murray PR, Baron EJ, Jorgensen JH, Landry ML, et al. Aspergillus, Fusarium, and other opportunistic moniliaceous fungi. Manual Clin Microbiol. 2006;2(9):1802-38.

4. Ben-Ami R, Lewis RE, Kontoyiannis DP. Enemy of the (immunosuppressed) state: an update on the pathogenesis of Aspergillus fumigatus infection. BrJHaematol. 2010;150(4):406-17.

5. Hope WW, Walsh TJ, Denning DW. Laboratory diagnosis of invasive aspergillosis. Lancet Infect Dis. 2005;5(10):609-22.

6. Sulahian A, Boutboul F, Ribaud P, Leblanc T, Lacroix C, Derouin F. Value of antigen detection using an enzyme immunoassay in the diagnosis and prediction of invasive aspergillosis in two adult and pediatric hematology units during a 4-year prospective study. Cancer. 2001;91(2):311-8.

7. Almeida IC, Neville DCA, Mehlert A, Treumann A, Ferguson AJ, Previato JO, et al. Structure of the N-linked oligosaccharide of the main diagnostic antigen of the pathogenic fungus Paracoccidiodes brasiliensis. Glycobiology. 1996;6(5):507-15.

8. Latge JP, Kobayashi H, Debeaupuis JP, Diaquin M, Sarfati J, Wieruszeski JM, et al. Chemical and immunological characterization of the extracellular galactomannan of Aspergillus fumigatus. Infect Immun. 1994;62(12):5424-33.

9. Morelle W, Bernard M, Debeaupuis JP, Buitrago M, Tabouret M, Latgé JP. Galactomannoproteins of Aspergillus fumigatus. $\mathrm{Eu}$ karyotic cell. 2005;4(7):1308-16.

10. Shahhosseini N. The spectrum of diseases caused by Aspergillus fumigatus. Iran J Clin Infect Dis. 2012;6(3):136-41.

11. Chan C, Woo PCY, Leung ASP, Lau SKP, Che X, Cao L, et al. Detection of antibodies specific to an antigenic cell wall galactomannoprotein for serodiagnosis of Aspergillus fumigatus aspergillosis. J Clin Microbiol. 2002;40(6):2041-5.

12. Fontaine T, Simenel C, Dubreucq G, Adam O, Delepierre M, Lemoine J, et al. Molecular organization of the alkali-insoluble fraction ofAspergillus fumigatus cell wall. J Biological Chem. 2000;275(36):27594-607.

13. Araújo FR, Melo VSP, Ramos CAN, Madruga CR, Soares CO, Kessler $\mathrm{RH}$, et al. Development of enzyme-linked immunosorbent assays based on recombinant MSP1a and MSP2 of Anaplasma marginale. Memórias do Instituto Oswaldo Cruz. 2005;100(7):765-69. 